\title{
Experience with alternative sources of structural bone graft for total wrist fusion in three rheumatoid arthritis patients
}

\author{
Richard A Hopper MD MSc ${ }^{1}$, Earl R Bogoch MD MSc FRCSC ${ }^{2}$ \\ ${ }^{1}$ Division of Plastic Surgery and ${ }^{2}$ Division of Orthopaedic Surgery, Department of Surgery, University of \\ Toronto, Toronto, Ontario
}

\begin{abstract}
RA Hopper, ER Bogoch. Experience with alternative sources of structural bone graft for total wrist fusion in three rheumatoid arthritis patients. Can J Plast Surg 2000;8(4):151-155.
\end{abstract}

Three cases are presented in which alternative sources of bone graft were used for wrist fusion; in two cases, frozen irradiated allograft was implanted, and in one, stored frozen autogenous bone harvested from the patient during a previous procedure was used. All cases were followed clinically and radiologically for at least one year. The frozen banked allograft and autograft were not successful when used as structural grafts to bridge a defect for wrist fusion; however, allograft implantation resulted in successful wrist fusion where host bone contact across the fusion site was maintained, and the allograft was used to fill a defect. Fresh autogenous bone remains the structural graft of choice for wrist fusion in the rheumatoid patient in the few cases where graft is required for this diagnosis. Morsellized frozen allograft can be employed to fill dead space.

Key Words: Allograft; Arthritis; Autograft; Fusion; Wrist

\section{Expérience avec d'autres sources de greffons osseux structuraux pour réaliser une arthrodèse complète du poignet chez trois patients atteints de polyarthrite rhumatoïde}

RÉSUMÉ: On présente trois cas pour lesquels on a eu recours à d'autres sources de greffons osseux pour réaliser une arthrodèse du poignet. Dans deux cas, on a implanté un allogreffon irradié et congelé, et dans un cas, on a utilisé de l'os autogène congelé et conservé en banque après son prélèvement chez un patient lors d'une intervention antérieure. Tous les cas ont fait l'objet d'un suivi clinique et radiologique pendant au moins un an. L'autogreffon et l'allogreffon congelés et conservés en banque, lorsqu'utilisés comme greffons structuraux, ne sont pas parvenus à combler une perte de substance osseuse pour réussir une arthrodèse du poignet ; cependant, l'implantation d'un allogreffon a permis une arthrodèse du poignet où le contact de l'os hôte à travers le site de fusion a été conservé, et où l'allogreffon a été utilisé pour combler une perte de substance. L'os autogène frais demeure le greffon structural de choix pour pratiquer une arthrodèse du poignet chez le patient atteint de polyarthrite rhumatoïde dans les quelques cas où la greffe est rendue nécessaire par le diagnostic. On peut utiliser des allogreffons congelés et en plusieurs morceaux pour combler l'espace mort.

$\mathrm{T}$ otal wrist arthrodesis provides a stable, painless and relatively functional hand for rheumatoid arthritis (RA) patients with severe carpal damage not responsive to lesser medical or surgical procedures (1-3). The majority of total wrist fusions in RA can be performed without the need for bone graft; however, for the uncommon case of a wrist with very severe bone resorption and instability, a structural graft may be required to restore carpal height, and maximize grasp and pinch strength. The standard graft employed in these cases is autogenous iliac crest bone harvested intraoperatively $(4,5)$.

Correspondence and reprints: Earl R Bogoch, Division of Orthopaedic Surgery, St Michael's Hospital - Wellesley Central Site, JB 434 - 160 Wellesley Street East, Toronto, Ontario M4Y 1J3. Telephone 416-926-4880, fax 416-926-5051, e-mail bogoch@istar.ca 

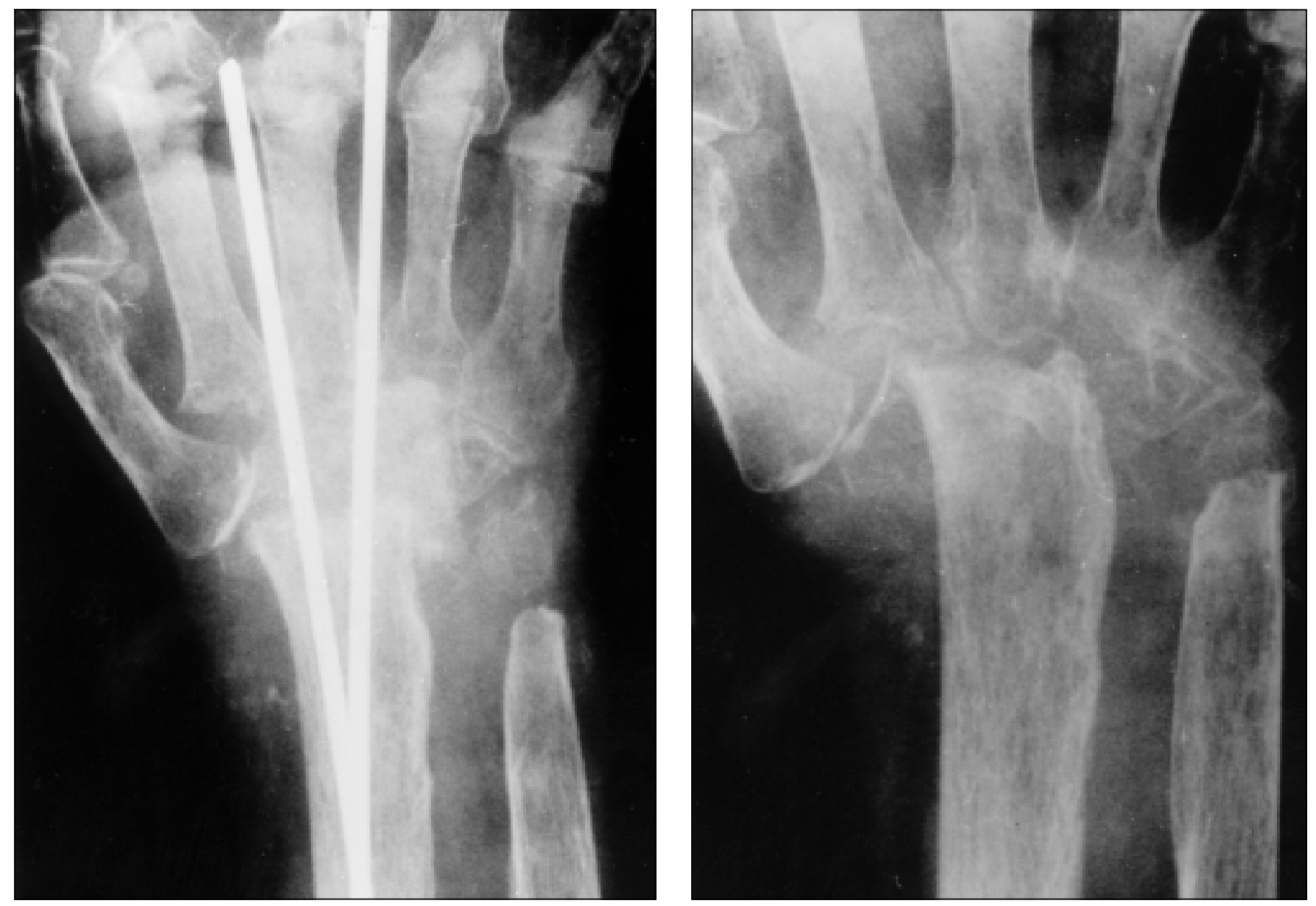

Figure 1) Left Early postoperative radiograph of the right wrist of a 57-year-old woman with severe polyarticular rheumatoid arthritis. A structural block of proximal femoral allograft is held in position with two Steinmann pins to provide stability and carpal height. Right The same wrist three months postoperation with the Steinmann pins removed. The femoral allograft has completely resorbed, and the wrist has returned to the preoperative state

Although very successful in providing a stable fusion, this bone source may be associated with donor site morbidity and may previously have been harvested during another surgical procedure (6-9).

We present three cases in which we employed bone other than fresh autogenous graft harvested intraoperatively. In two cases, frozen irradiated allograft was used, once to restore carpal height and once to fill dead space in the presence of partial host bone contact. In another patient, stored frozen autogenous bone harvested during a previous procedure was used as a structural graft.

\section{Case 1}

\section{CASE PRESENTATIONS}

A 57-year-old woman had a 25-year history of severe arthritis mutilans involving both wrists, hands, shoulders, hips, knees and ankles, as well as the cervical spine and lungs. She had undergone multiple surgical procedures including bilateral hip arthroplasty, cervical and subtalar fusions, and Swanson arthroplasty of the hand. She presented with a completely unstable right wrist, which could be pistoned and telescoped axially, and translated several centimetres in both the sagittal and coronal planes. Preoperative radiographs demonstrated virtually complete destruction of the carpal bones, and severe erosion of the distal radius and ulna and metacarpal bases. She was admitted for arthrodesis of the right wrist with major allograft reconstruction, as well as for right pantalar arthrodesis. The decision to use frozen allograft in both procedures was made due to the large amount of graft that would be required, the history of past iliac crest harvesting and the patient's wish to avoid delay in postoperative mobilization due to donor site pain. Following debridement of the right wrist through a dorsal approach, a block of proximal femoral allograft from the hospital bone bank was slotted into the radius to restore $6 \mathrm{~cm}$ of carpal height. The allograft, harvested from a donor who had undergone total hip arthroplasty, had been irradiated with $2.5 \mathrm{Megarads}$, then stored at $-80^{\circ} \mathrm{C}$. The fusion was stabilized with two Steinmann pins passed between the intermetacarpal spaces, through the allograft and into the distal radius (Figure 1, left). Fixation was felt to be precarious due to the fragility and poor quality of the bone. Extensor tenosynovectomy and tendon transfer of extensor carpi radialis longus to extensor pollicis longus were performed at the same time.

Two months later, one stabilization wire was removed due to protrusion and pain. Three months postoperatively, the 

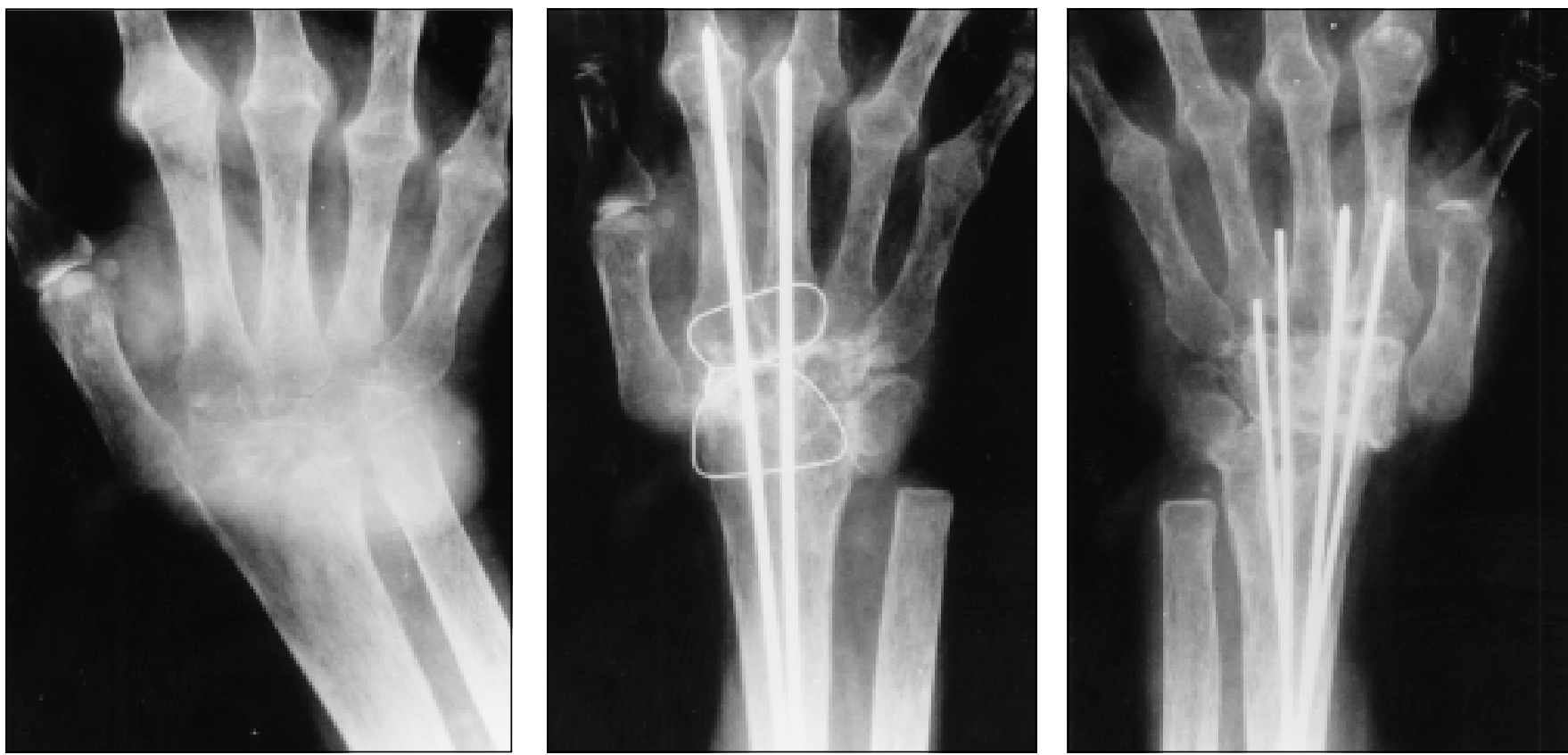

Figure 2) Left Preoperative radiograph of the right wrist of a 36-year-old woman with rheumatoid arthritis. There is severe resorption of the carpal bones, the distal radius and the ulna. Middle Three years following carpal debridement and bone grafting with large fragments of femoral head allograft, the wrist appears to be fused in good position. It was not necessary to remove the two Steinmann pins. Right Radiograph of the contralateral wrist of the same patient, one year following wrist fusion. The structural block of fresh iliac crest bone graft appears to be incorporated, and the wrist is fused in a satisfactory position

final wire was removed and the wrist was clinically confirmed to be unfused, with unstable dislocation of the wrist. On the radiograph of the wrist, the allograft was seen to be completely resorbed (Figure 1, Right). Revision of the fusion using fresh iliac autograft was recommended and is planned.

\section{Case 2}

A 36-year-old, morbidly obese woman with a 14-year history of polyarticular RA was referred by the rheumatology service with a one-year history of pain that worsened with movement on the ulnar side of her wrist, as well as an inability to extend the fingers of her right hand. She had been treated with multiple medications, including predisone, and had undergone bilateral total hip arthroplasty. Clinical and radiological examination revealed bone resorption and dislocation of both wrists, with caput ulnae syndrome, extensor tenosynovitis and rupture of the majority of her extensor tendons (Figure 2, Left). Under general anesthesia and through a dorsal approach, an extensor tenosynovectomy and carpal debridement were performed. The wrist was stabilized with two Steinmann wires through the metacarpals and carpals, and passed into the radius. Although ventral bony contact existed from the radius through remnants of carpal bones to the bases of the index and middle metacarpals, large bony gaps remained around the fusion site. Some of these gaps were partially filled by morsellized autogenous bone excised intraoperatively from the distal ulna. The majority of the dead space, however, was filled with large fragments of femoral head allograft from the hospital bone bank. A figure-of-eight wire was placed across the fusion site to minimize the axial instability caused by the ligamentous laxity and tendon destruction. A tendon graft of palmaris longus and half of extensor carpi ulnaris was used to reconstruct the digital extensor mechanism. The extensor carpi ulnaris tendon was also used to perform a tenodesis to stabilize the distal ulna.

On last follow-up, 36 months postoperatively, the patient's wrist was pain free and stable in a neutral position. Full forearm supination and pronation were demonstrated, and the patient reported a moderate improvement in hand function. On the wrist radiograph, the Steinmann pins were still in place and the wrist appeared fused (Figure 2, Middle). Two years following the right wrist fusion, the left wrist, which demonstrated more severe carpal resorption and instability, was fused using a structural block of fresh autogenous iliac crest and four Steinmann pins. At one-year follow-up, the left wrist was solidly fused with maintenance of carpal height (Figure 2, Right).

\section{Case 3}

A 39-year-old man with a sixteen-year history of severe polyarticular RA was admitted for surgical correction of a clinically unstable left wrist. The joint had been treated 18 months previously by an attempted arthrodesis without bone graft that failed when the stabilizing Steinmann pins inserted between the metacarpal heads had to be removed. Following this operation, the wrist returned to its preoperative state, with further resorption of the carpal bones and bases of the metacarpal bones, resulting in complete wrist instability and hand dysfunction. Radiographs showed severe destructive changes involving the radiocarpal and intercarpal joints, with distal ulnar erosion and almost complete loss of carpal height. An autogenous tricortical iliac crest bone graft had been har- 

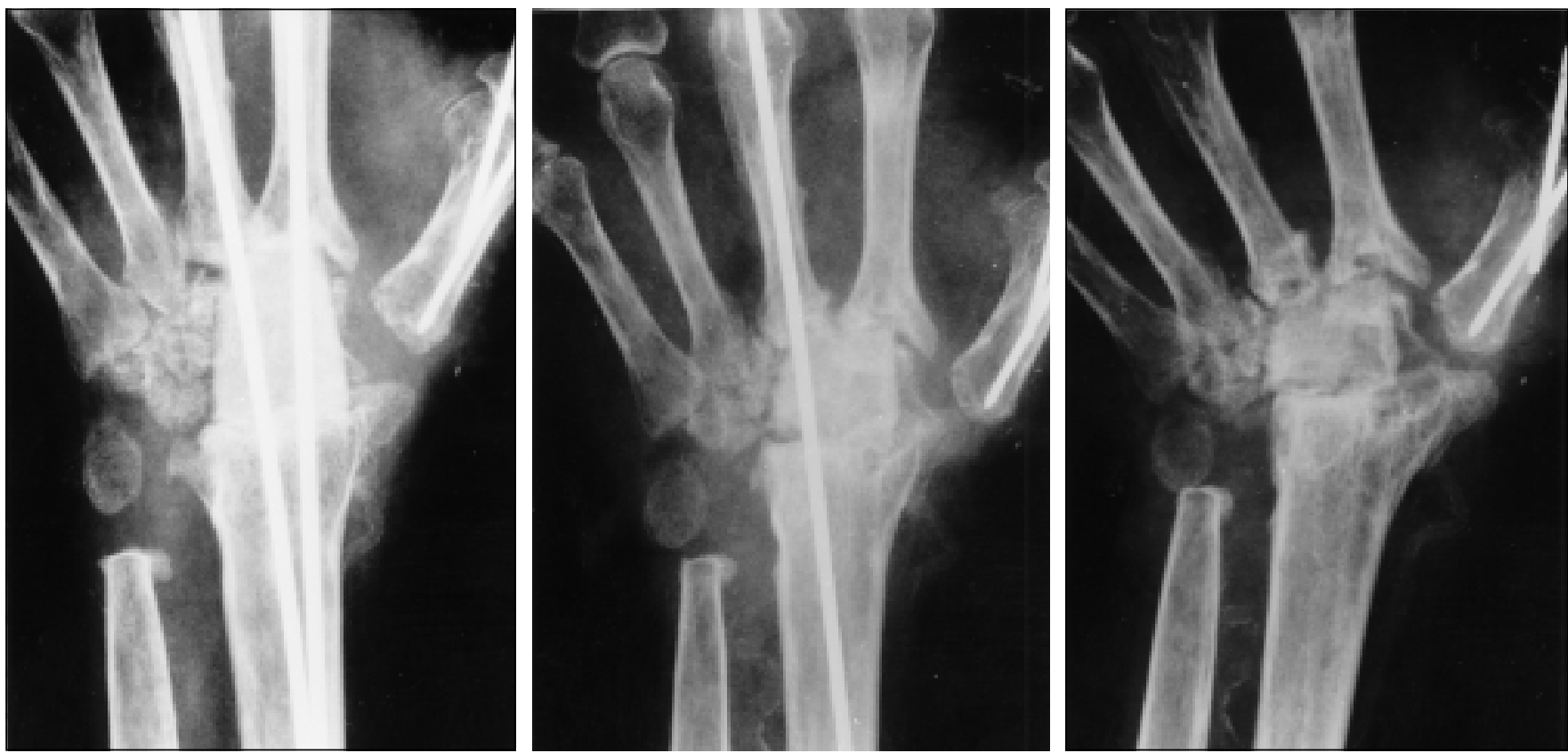

Figure 3) Left Early postoperative radiograph of the left wrist of a 39-year-old man with severe polyarticular rheumatoid arthritis. Fusion was performed with a frozen tricortical structural iliac crest bone graft harvested from the patient during an earlier surgery. The graft restored carpal height and placed the wrist in a more functional position. Middle Eight months postoperation, one Steinmann pin was removed due to pin migration and protrusion. The frozen iliac crest autograft demonstrated evidence of resorption with a $50 \%$ loss of vertical height. Right At one year postoperation, the second Steinmann pin was removed. The frozen iliac crest autograft progressively resorbed, with loss of wrist position

vested for an ankle fusion procedure four months earlier, and an unused portion had been frozen in the hospital's bone bank. The procedure was planned in consultation with the patient to avoid further donor site morbidity during anticipated future reconstructive surgery of the wrist.

Under general anesthesia, the patient underwent arthrodesis of the left wrist using the banked autograft. Through a dorsal wrist incision, the carpal bones, proximal metacarpals and distal radius were rongeured to expose healthy bone. The autograft was placed as a tricortical structural block between the distal radius and the bases of the index and middle metacarpal bones. The fusion was stabilized by Steinmann pins passed through the head of the metacarpals, the autograft and distal radius (Figure 3, left).

Eight months postoperatively, the Steinmann pins were removed due to partial extrusion. On radiograph, there was evidence of loss of longitudinal height of the bone graft (Figure 3 , middle). A scintigram of the wrist at that time revealed intense uptake at the proximal and distal junctions of the graft with host bone, and a zone of lesser radionuclide uptake in the centre of the graft. At one year postoperatively, there was clinical and radiographical evidence of nonunion of the graft, with progressive loss of carpal height (Figure 3, Right). The patient declined further surgical treatment of his wrist.

\section{DISCUSSION}

Patients with severe polyarticular RA frequently require multiple joint and soft tissue surgical procedures over a relatively short period of time. The risk of perioperative complications is increased in these individuals due to cardiopulmonary disease, impaired wound healing and decreased ability to ambulate. It is desirable to diminish the invasive nature of procedures and combine them while minimizing postoperative immobilization and loss of function. At our institution, the majority of rheumatoid wrist fusions do not require grafting of bone other than from local sources in the wrist. In those infrequent cases with severe resorption (loss of carpal height and instability that require more extensive grafting), autogenous iliac crest bone has been harvested intraoperatively. This, however, increases operative time, and can impair postoperative rehabilitation due to donor site pain and the risk of complications (6-9). In the three cases described, we attempted to avoid harvesting autogenous iliac bone for total wrist fusion by using either frozen irradiated banked allograft or frozen autograft banked during a previous procedure.

Anderson (10) described the successful use of frozen allograft in three types of reconstructive carpal surgery. However, in each case, the grafts employed were small, thin nonstructural pegs measured in millimetres. Clinical experience with large, frozen structural allografts outside the upper limb has generally not been highly reliable, with overall complication rates ranging from $27 \%$ to $70 \%$ (11-14). Szabo et al (15) reported the replacement of excised radial heads with size-matched frozen allograft; however, we were unable to find any reports in the literature of the use of structural allograft in fusion of the rheumatoid wrist. Recently, we learned that another surgeon's experience with structural allograft for bridging major gaps in fusion of the rheumatoid wrist has been similar to our own, with three of four cases failing to fuse (RD Beckenbaugh, personal communication).

In case 1, a block of proximal femoral cancellous allograft was used as a structural support; however, the wrist failed to 
fuse and the graft resorbed, with migration of the Steinmann pins and recurrent wrist instability. In case 2, the allograft was used in large fragments, with a small amount of locally harvested autograft used to fill dead space at the fusion site. If allograft had not been employed, harvesting autograft from the iliac crest would have been required. The fusion was successful, perhaps because there was contact of the host bone, and the allograft was not required for restoration and maintenance of carpal height.

The patient in case 3 was severely affected with RA and required multiple procedures. We decided to harvest additional iliac crest bone during an ankle fusion procedure and bank it in anticipation of future need for graft material in the wrist or elsewhere. Use of the frozen bone four months later for total wrist fusion was not successful after one-year followup, due to graft resorption and nonunion.

To our knowledge, the use of alternative sources of bone

\section{REFERENCES}

1. Carroll RE, Dick HM. Arthrodesis of the wrist for rheumatoid arthritis. J Bone Joint Surg [Am] 1971;53:1365-9.

2. Clayton ML, Ferlic DC. Arthrodesis of the arthritic wrist. Clin Orthop 1984;187:89-93.

3. Kobus RJ, Turner RH. Wrist arthrodesis for treatment of rheumatoid arthritis. J Hand Surg [Am] 1990;15:541-6.

4. Campbell CJ, Keokarn T. Total and subtotal arthrodesis of the wrist. J Bone Joint Surg [Am] 1964;46:1520-33.

5. Lenoble E, Ovadia H, Goutallier D. Wrist arthrodesis using an embedded iliac crest bone graft. J Hand Surg [Br] 1993;18:595-600.

6. Cockin J. Autologous bone grafting - complications at the donor site. J Bone Joint Surg [Br] 1971;53:153. (Abst)

7. Coventry MB, Tapper EM. Pelvic instability: a consequence of removing iliac bone for grafting. J Bone Joint Surg [Am] 1972;54:83-101.

8. Fromberg VG, Schmidt A, Ishida A. Vergleich der hebedefekte von fibula- und beckenkammtransplantaten. Handchi Mikrochir Plast Chir 1995;27:102-4.

9. Kuhn DA, Moreland MS. Complications following iliac crest bone grafting. Clin Orthop 1986;209:224-6. graft in the rheumatoid wrist has not previously been discussed in the literature. Given the small number of patients who received allograft or banked autograft for total wrist fusion at our institution, it is not possible to draw any firm conclusions about the success of these modalities compared with that of fresh iliac crest bone. In addition, differences in individual surgeon preferences for method of fixation and postoperative immobilization make it difficult to extrapolate our experience to other centres. Following our experience with these three cases, however, we decided not to use blocks of allograft or banked autograft when structural support is required. We have resumed the use of fresh structural iliac crest autograft in the uncommon case where a distracting interpositional block graft is necessary (case 2, Figure 2, right). In patients requiring filling of dead space at the wrist, but who have bony contact across the fusion zone, we will continue to use morsellized, frozen, irradiated, banked allograft when sufficient local autograft is not available.

10. Anderson WJ. Allograft bone for arthrodesis and repair of skeletal hand problems. J Hand Surg [Br] 1989;14:332-5.

11. Aho AJ, Ekfors T, Dean PB, Aro HT, Ahonen A, Nikkanen V. Incorporation and clinical results of large allografts of the extremities and pelvis. Clin Orthop 1994;307:200-13.

12. Gebhardt MC, Flugstad DI, Springfield DS, Mankin HJ. The use of bone allografts for limb salvage in high-grade extremity osteosarcoma. Clin Orthop 1991;270:181-96.

13. Mankin HJ, Gebhardt MC, Tomford WW. The use of frozen cadaveric allografts in the management of patients with bone tumors of the extremities. Orthop Clin North Am 1987;18:275-89.

14. Mnaymneh W, Malinin TI, Makley JT, Dick HM. Massive osteoarticular allografts in the reconstruction of extremities following resection of tumors not requiring chemotherapy and radiation. Clin Orthop 1985;197:76-87.

15. Szabo RM, Hotchkiss RN, Slater RR Jr. The use of frozen-allograft radial head replacement for treatment of established symptomatic proximal translation of the radius: preliminary experience in five cases. J Hand Surg [Am] 1997;22:269-78. 